\title{
PROPIEDADES PSICOMÉTRICAS DE LA ESCALA DE FUNCIONALIDAD FAMILIAR FACES-III: UN ESTUDIO EN ADOLESCENTES PERUANOS
}

\author{
Juan Carlos Bazo-Alvarez, ${ }^{1,2, a}$, Oscar Alfredo Bazo-Alvarez ${ }^{2,3, b}$, Jeins Aguila ${ }^{2, a}$, Frank Peralta ${ }^{2, c}$, \\ Wilfredo Mormontoy ${ }^{1, d}$, Ian M. Bennett ${ }^{4, e}$
}

\begin{abstract}
RESUMEN
Objetivos. Evaluar las propiedades psicométricas del FACES-III en una población de estudiantes adolescentes peruanos. Materiales y métodos. El presente estudio es transversal, de tipo psicométrico. El muestreo aplicado fue probabilístico, definido en tres etapas: primer estrato (colegio), segundo estrato (grado), conglomerado (sección). Los participantes fueron 910 estudiantes adolescentes de ambos sexos, entre los 11 y 18 años. El instrumento es, a la vez, el objeto de estudio: el FACES-III de Olson. El análisis incluyó la revisión de la validez de estructura/constructo mediante análisis factorial y de la confiabilidad por consistencia interna (índices alpha, theta y omega). Resultados. La escala cohesiónreal tiene confiabilidad moderadamente alta $(\Omega=0,85)$ mientras que la escala flexibilidad-real tiene confiabilidad moderada $(\Omega=0,74)$. Para la escala cohesión-ideal la confiabilidad es moderadamente alta $(\Omega=0,89)$, lo mismo que para la escala de flexibilidad-ideal $(\Omega=0,86)$. La validez de constructo se confirmó en la bondad de ajuste del modelo de dos factores (cohesión y flexibilidad) con 10 ítems cada uno [índice ajustado de bondad de ajuste (AGFI)=0,96; índice de validación cruzada esperada $(\mathrm{ECVI})=0,87$; índice de ajuste normado $(\mathrm{NFI})=0,93$; índice de bondad de ajuste $(\mathrm{GFI})=0,97$; raíz del error cuadrático medio de aproximación (RMSEA) $=0,06]$. Conclusiones. EI FACES-III tiene confiabilidad y validez suficientes como para ser utilizado en población adolescente peruana con fines de evaluación grupal o individual.
\end{abstract}

Palabras clave: Relaciones familiares; Análisis factorial; Propiedades psicométricas (fuente: DeCS BIREME).

\section{PSYCHOMETRIC PROPERTIES OF THE THIRD VERSION OF FAMILY ADAPTABILITY AND COHESION EVALUATION SCALES (FACES-III): A STUDY OF PERUVIAN ADOLESCENTS}

\begin{abstract}
Objetives. Our aim was to evaluate the psychometric properties of the FACES-III among Peruvian high school students. Materials and Methods. This is a psychometric cross-sectional study. A probabilistic sampling was applied, defined by three stages: stratum one (school), stratum two (grade) and cluster (section). The participants were 910 adolescent students of both sexes, between 11 and 18 years of age. The instrument was also the object of study: the Olson's FACES-III. The analysis included a review of the structure / construct validity of the measure by factor analysis and assessment of internal consistency (reliability). Results. The real-cohesion scale had moderately high reliability $(\Omega=.85)$ while the real-flexibility scale had moderate reliability $(\Omega=.74)$. The reliability found for the ideal-cohesion was moderately high $(\Omega=.89)$ like for the scale of ideal-flexibility $(\Omega=.86)$. Construct validity was confirmed by the goodness of fit of a two factor model (cohesion and flexibility) with 10 items each [Adjusted goodness of fit index $(\mathrm{AGFI})=0.96$; Expected Cross Validation Index $(\mathrm{ECVI})=0.87$; Normed fit index $(\mathrm{NFI})=0.93$; Goodness of fit index $(\mathrm{GFI})=0.97$; Root mean square error of approximation $(\mathrm{RMSEA})=$ 0.06]. Conclusions. FACES-III has sufficient reliability and validity to be used in Peruvian adolescents for the purpose of group or individual assessment.
\end{abstract}

Key words: Family relations; Factorial analysis; statistical; Psychometric properties. (source: MeSH NLM).

\footnotetext{
Departamento de Estadística, Demografía, Humanidades y Ciencias Sociales, Facultad de Ciencias y Filosofía, Universidad Peruana Cayetano Heredia. Lima, Perú.

2 PSYCOPERU - Instituto de Investigación y Capacitación para la Prevención y Promoción Psicoeducativa y Psicosocial. Lima, Perú.

3 Escuela de Medicina Humana, Facultad de Ciencias de la Salud, Universidad Privada San Juan Bautista. Lima, Perú.

4 Department of Family Medicine, School of Medicine, University of Washington. EE. UU.

a Licenciado en Psicología; ${ }^{b}$ médico cirujano; ' licenciado en Biología; ${ }^{\mathrm{d}}$ maestro en Salud Pública; ${ }^{\mathrm{e}}$ Doctor of Philosophy.

Recibido: 02/11/2015 Aprobado: 01/06/2016
}

Citar como: Bazo-Alvarez JC, Bazo-Alvarez OA, Aguila J, Peralta F, Mormontoy W, Bennett IM. Propiedades psicométricas de la escala de funcionalidad familiar FACES-III: un estudio en adolescentes peruanos. Rev Peru Med Exp Salud Publica. 2016;33(3):462-70. doi: 10.17843/rpmesp.2016.333.2299 


\section{INTRODUCCIÓN}

La funcionalidad familiar es un fenómeno siempre complejo y difícil de evaluar, especialmente con instrumentos psicométricos. Las primeras fórmulas no cuantitativas de evaluación incluían (y aún lo hacen) entrevistas personales a profundidad con cada miembro (por separado) o entrevistas circulares con todos los miembros (en simultáneo). Estas soluciones implicaban una inversión muy grande de tiempo y esfuerzo, para todas las partes. Luego, especialistas en el funcionamiento de la pareja y la familia comenzaron a estandarizar procedimientos e instrumentos que permitieran realizar estas evaluaciones, aunque de forma más sencilla (instrumentos estandarizados). De esta manera, se fueron gestando una serie de instrumentos alusivos: FACES-I ${ }^{(1)}$, FACES-II (2), FACESIII ${ }^{(3,4)}$ y el FACES-IV ${ }^{(5,6)}$. La introducción al medio de estas versiones vino acompañada de un modelo teórico que iría madurando en el tiempo, para facilitar el estudio y medición de la funcionalidad familiar: el modelo circumplejo ${ }^{(7,8)}$.

El modelo circumplejo de Olson propone tres dimensiones para explicar la funcionalidad familiar: cohesión, flexibilidad y comunicación ${ }^{(9)}$. La cohesión hace referencia al grado de unión afectiva entre miembros de una familia. La flexibilidad habla sobre el mundo de las normas y el liderazgo en el hogar. La comunicación opera como una dimensión facilitadora. Sin embargo, en la propuesta del FACES-III, solo se trabaja directamente con la cohesión y la flexibilidad, cada una medida en cuatro niveles. Los niveles extremos de cualquiera de ellas (el más bajo o el más alto) se consideran disfuncionales, y los niveles medios se los reconoce como funcionales. Así, cada dimensión de la funcionalidad forma uno de los dos ejes de un sistema cartesiano que permite clasificar hasta en dieciséis tipos de familias (Figura 1). Estos tipos familiares se ubican dentro de "rangos" que les asignan algún nivel específico de funcionalidad familiar: balanceadas (o funcionales), rango medio y extremas (o disfuncionales).

Sin embargo, el constructo funcionalidad medido en forma bidimensional (FACES-III), no ha mostrado similar validez en todas las culturas: la flexibilidad parece descomponerse en más de una faceta. En España, se identificaron y postularon hasta seis dimensiones ${ }^{(10)}$, mientras que otros reconocieron hasta tres ${ }^{(11)}$. En Argentina, se propuso la solución de tres factores como superior a la de dos ${ }^{(12)}$, e incluso se sugirió la eliminación de algunos ítems. En México, avalaron un modelo de cuatro factores ${ }^{(13)}$. En Chile, se encontró evidencia de hasta siete factores, aunque se optó por una solución de segundo orden ${ }^{(14)}$. En Suiza, la

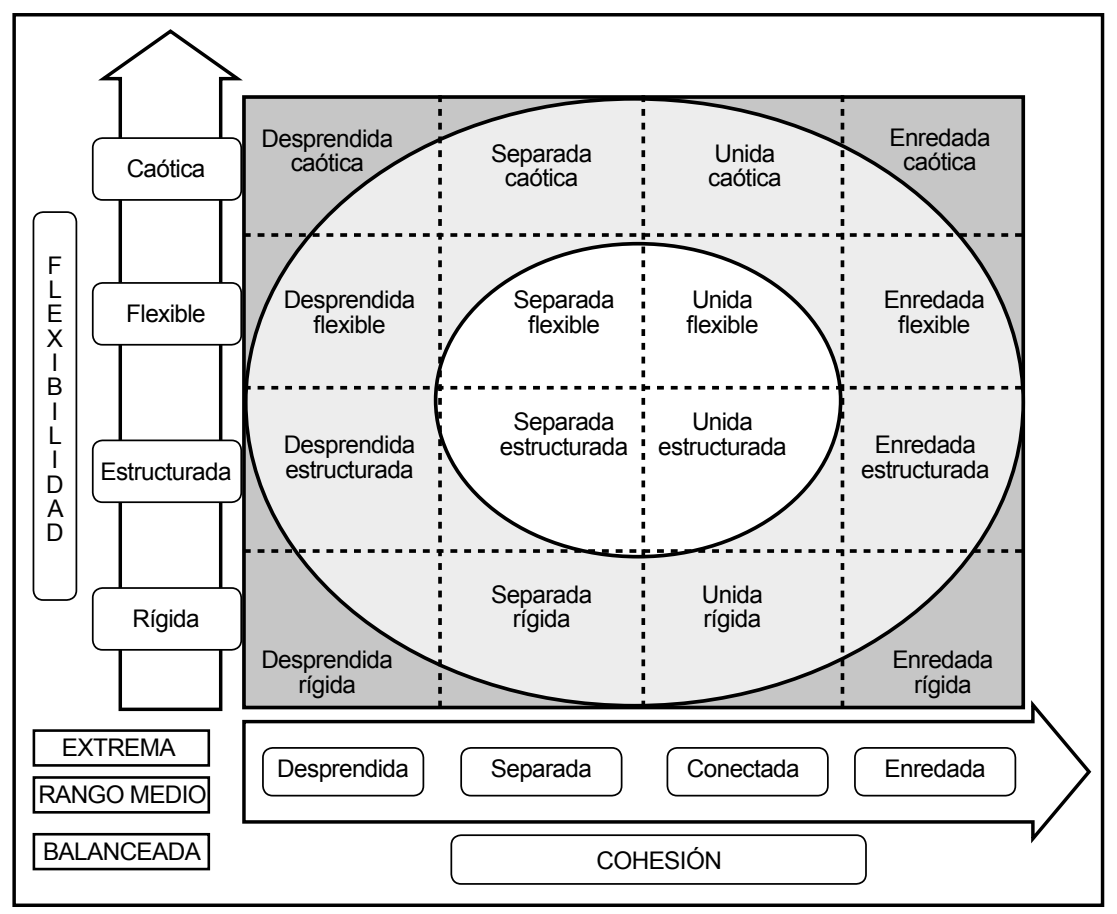

Fuente: elaboración propia, basado en el manual original del FACES-III de Olson, Portner y Lavee (1985).

Figura 1. Modelo circumplejo para el FACES-III de Olson. Los ejes representan las dimensiones de cohesión y flexibilidad y a partir de ellos se genera la clasificación de dieciséis tipos familiares 
propuesta de dos dimensiones resultaba verosímil, pero tras la eliminación de ciertos ítems ${ }^{(15)}$. De hecho, esta gran variedad entre resultados es de esperar si se considera que la funcionalidad familiar está muy ligada a la cultura que enmarca a la familia.

Lo anterior supone la primera de varias razones que justifican el realizar un estudio como este en Perú. La variabilidad del dimensionamiento de la funcionalidad familiar a través de distintas culturas exige saber qué ocurre concretamente en nuestro escenario local con el FACES-III. Sin esta información, nuestro uso e interpretación de su medida puede estarse realizando de manera incorrecta. Más allá, el uso generalizado actual de este instrumento, tanto para evaluación como para investigación (especialmente en la costa norte del Perú), hacen de esta una necesidad urgente.

En este sentido, el objetivo del presente estudio ha sido realizar una revisión de las propiedades psicométricas del FACES-III de Olson en una población de estudiantes adolescentes peruanos. En concreto, hemos revisado la evidencia de confiabilidad y de validez de constructo, verificando si la solución bidimensional (flexibilidad/ cohesión) es verosímil para nuestra población.

\section{MATERIALES Y MÉTODOS}

\section{DISEÑO}

El presente estudio es transversal, de tipo psicométrico. El diseño contempla el desarrollo de dos acciones específicas: i) la revisión de la confiabilidad a través de un análisis de consistencia interna; y ii) la revisión de la validez a través de un análisis de la estructura/ constructo del test.

\section{POBLACIÓN Y MUESTRA}

La población objetivo se definió como todos los estudiantes de secundaria matriculados en las instituciones educativas privadas y nacionales ubicadas en los distritos de Chimbote y Nuevo Chimbote, durante el año académico 2015. La población fuente estuvo definida como todos los estudiantes de secundaria matriculados en dos instituciones educativas, una privada y otra nacional, pertenecientes a la población objetivo. Para el caso de la institución educativa privada, se incluyó también al sexto grado de primaria (a solicitud de dicha institución). El único criterio de inclusión fue estar matriculado en la institución educativa (IE) privada o la IE nacional elegidas. Se manejaron dos criterios de exclusión: i) rehusarse a responder voluntariamente la encuesta del estudio, y ii) brindar menos del $90 \%$ de toda la información solicitada.
El muestreo fue probabilístico, definido en tres etapas: institución educativa como primer estrato (nacional y particular), grado de estudio como segundo estrato y secciones del grado de estudio como conglomerados (unidades primarias de muestreo). Para los estratos, la afijación fue proporcional. Los conglomerados fueron seleccionados aleatoriamente y sin reposición, dentro de cada estrato, hasta garantizar que se cubriera la cuota muestral del estrato. Cada conglomerado seleccionado fue censado en su totalidad. El marco muestral fue de 53 salones de clase (conglomerados) con un total de 1957 alumnos. La unidad de análisis fue una aplicación de la Escala de Funcionalidad Familiar FACES-III de Olson respondida por un estudiante de la muestra. Se calcularon los pesos muestrales a partir de la información de este diseño muestral, utilizando las probabilidades de inclusión para cada conglomerado/estrato (el peso es igual al producto de los inversos de la probabilidad de cada stage). También se determinó una corrección de población finita (FPC) para muestreo sin reposición.

Para el tamaño de la muestra se consideró un margen mínimo según las recomendaciones para análisis factorial exploratorio y confirmatorio (16-18), aplicando el método de Kim mediante una rutina SPSS 20, cuyas fórmulas pueden verse en las referencias (19-22). Adicionalmente, se consideró un ajuste por pérdida de muestra de $10 \%$. La configuración de la muestra puede verse en el Anexo 1 (material suplementario), con un tamaño total de 910 estudiantes.

\section{INSTRUMENTO}

El instrumento es la prueba psicométrica objeto de estudio del presente. Se utilizó la escala de evaluación de la cohesión y adaptabilidad familiar, tercera versión o FACES-III ${ }^{(4)}$, adaptada a la población española ${ }^{(14,23)}$. EI FACES-III evalúa funcionalidad familiar a partir de la medición de dichas dimensiones del modelo circumplejo. Es un instrumento de autorreporte que consta de 20 ítems con cinco opciones de respuesta en formato Likert (nunca o casi nunca; pocas veces; algunas veces; frecuentemente; y siempre o casi siempre). EI FACESIII tiene dos formatos, uno para familia real, que enfoca el análisis de los ítems bajo la percepción actual que posee el participante sobre su dinámica familiar; y otro para familia ideal, que se enfoca en cómo se desearía que funcione la propia familia. El formato de familiar real fue aplicado a los 910 estudiantes, el de familia ideal a una submuestra de 100 de los 910 .

\section{VARIABLES DE ESTUDIO}

La cohesión familiar se interpreta en cuatro niveles: familia desprendida: primacía del 'yo', ausencia de unión afectiva entre los familiares, ausencia de lealtad a la familia y alta independencia personal; familia separada: 
primacía del 'yo' con presencia del 'nosotros', moderada unión afectiva entre los familiares, cierta lealtad e interdependencia entre los miembros de la familia, aunque con un cierto sesgo hacia la independencia; familia unida: primacía del 'nosotros' con presencia del 'yo', considerable unión afectiva entre los familiares, cierta lealtad, fidelidad e interdependencia entre los miembros de la familia, aunque con algún sesgo hacia la independencia, y familia enredada o amalgamada: primacía del 'nosotros', máxima unión afectiva entre los familiares, exigencia de fidelidad y lealtad a la familia, y alto grado de dependencia a las decisiones tomadas en común ${ }^{(10)}$. Olson postuló que los niveles balanceados (separada y unida) hacen un funcionamiento familiar óptimo y sus miembros son capaces de ser independientes y conectados a sus familias. Los niveles desbalanceados (desprendida y amalgamada) son vistos como problemáticos (o disfuncionales), si se actúa en estos niveles extremos durante mucho tiempo ${ }^{(9)}$

La flexibilidad familiar se interpreta en cuatro niveles: familia caótica: ausencia de liderazgo, cambios aleatorios y/o excesivos de roles, disciplina irregular; familia flexible: liderazgo compartido, roles compartidos, disciplina democrática y cambios cuando son necesarios; familia estructurada: liderazgo a veces compartido, roles en ocasiones compartidos, cierto grado de disciplina democrática y los cambios ocurren cuando se solicitan; familia rígida: liderazgo autoritario, roles fijos, disciplina estricta y ausencia de cambios ${ }^{(10)}$. Los niveles extremos de flexibilidad (caótica y rígida) tienden a ser problemáticos, y los niveles moderados (flexible y estructurado) tienden a balancear cambio y estabilidad en un modo más funcional.

La matriz de operacionalización construida por los autores y visible en el material suplementario (Anexo 2) puede ayudar a integrar los conceptos antes descritos con la medición realizada por el FACES-III. Las variables atributivas relevantes al estudio son la edad, el sexo, la institución educativa y el grado de estudios. Los valores para estas variables fueron los declarados en el cuestionario sociodemográfico del estudio.

\section{PROCESAMIENTO Y ANÁLISIS ESTADÍSTICO}

Se realizó un análisis de valores perdidos que incluyó la verificación del supuesto de aleatoriedad y un tratamiento mediante el método de imputación simple por regresión estocástica. Dicho método consiste en imputar cada valor perdido con el resultado estocástico (outcome) de un modelo de regresión que tiene como predictores a todos los demás ítems de una misma escala (cohesión y flexibilidad por separado). Esta imputación tiene el propósito de reducir el error de las estimaciones que deviene de la pérdida de datos que estaban contemplados en el tamaño muestral calculado. Posteriormente, se desarrolló el análisis exploratorio de datos, el cual incluyó la revisión de las características de la muestra, distribución de frecuencias y detección de outliers.

La confiabilidad se estimó en base a la consistencia interna. En tal sentido, se realizó una revisión de homogeneidad mediante matrices de correlación ítem-ítem e ítem-resto, en forma simultánea con el alfa de Cronbach, para cada escala por separado. Adicionalmente, se calcularon los índices omega y theta, los cuales son más apropiados para evaluar la consistencia interna de instrumentos multidimensionales ${ }^{(24)}$.

La validez se verificó mediante análisis factoriales. Primero, y tras verificar los supuestos, se procedió con el análisis factorial exploratorio (AFE) a nivel de cada escala por separado (cohesión y flexibilidad). Dicho AFE se realizó a partir de matrices policóricas, utilizando el método de factores principales y la rotación Oblimin. Este AFE permitió evaluar la unidimensionalidad de cada una de estas escalas. Para verificar la validez de la estructura bidimensional original del FACESIII se realizó un análisis factorial confirmatorio (AFC) con el método de mínimos cuadrados ponderados robustos ${ }^{(25)}$, a nivel del instrumento global (escalas de cohesión y flexibilidad evaluadas en simultáneo). De esta manera, se contrastaron hasta seis modelos bidimensionales diferenciados entre sí solo por la eliminación progresiva de ítems heterogéneos (a los que se denominó "truncados"). Estos modelos truncados mantenían las dos dimensiones (cohesión y flexibilidad), pero en ellos se fueron eliminando paulatinamente los ítems menos homogéneos de la escala de flexibilidad. Este procedimiento llevó a evaluar hasta seis (6) nuevos modelos truncados, los mismos que tenían en común una dimensión de cohesión con sus diez (10) reactivos completos, pero diferían en los ítems que se conservaban en la dimensión flexibilidad. Estos modelos truncados fueron comparados entre sí en función a su bondad de ajuste, utilizando indicadores conocidos (26): índice ajustado de bondad de ajuste (AGFI), índice de validación cruzada esperada (ECVI), raíz del error cuadrático medio de aproximación (RMSEA) y chi cuadrado escalada de Satorra-Bentler.

Todo el análisis estadístico se desarrolló con los paquetes Stata 12, SPSS 20, LISREL 8.1, AMOS 18 y Excel. 


\section{ASPECTOS ÉTICOS}

Este estudio cuenta con la aprobación del Comité de Ética de PSYCOPERU y del Comité de Ética de la Universidad Peruana Cayetano Heredia, y cumple con los principios éticos en investigación contemplados en el Código de Ética de Psicólogos del Perú, en el de la American Psychological Association y en la Declaración de Helsinki.

\section{RESULTADOS}

La muestra tuvo las siguientes características: i) en la IE nacional hubo $47,9 \%$ de mujeres, con una edad media de 14,2 \pm 1,5 D.E. (desviación estándar); ii) en la IE particular hubo $62,0 \%$ de mujeres, con una edad media de 13,5 (D.E. $\pm 1,8$ ). Un pequeño porcentaje de respuestas faltantes a los ítems fue imputado: $1 \%$ general y no más de 2,3\% por ítem para la escala real del FACES-III $(\mathrm{N}=910)$ y $1,3 \%$ general y no más de $7 \%$ por ítem para la escala ideal $(\mathrm{N}=100)$. Para todas las escalas se confirmó el supuesto de aleatoriedad (Missing at Random o MAR). Tras la imputación simple por regresión estocástica se verificó que el impacto de dicha imputación no afectó las medias y desviaciones estándar de los ítems, tal y como puede verse en las Anexos 3 y 4 (material suplementario).

El análisis con matrices de correlaciones inter-ítem e ítem-resto, mostró niveles moderados a altos de homogeneidad para las escalas reales e ideales. La escala de cohesión real $(\mathrm{N}=910)$ mostró correlaciones policóricas entre 0,10 y 0,60 (media 0,32 y D.E. 0,14 ), mientras la escala de cohesión ideal $(\mathrm{N}=100)$ mostró correlaciones policóricas entre 0,14 y 0,66 (media 0,45 y D.E. 0,13 ). Las correlaciones ítem-resto para la escala de cohesión real estuvieron entre 0,19 y 0,63 (media 0,47 y D.E. 0,14$)$, mientras que para la escala de cohesión ideal estuvieron entre 0,38 y 0,72 (media 0,55 y D.E. 0,10 ). La escala de flexibilidad real $(\mathrm{N}=910)$ mostró correlaciones policóricas entre $-0,08$ y 0,41 (media 0,12 y D.E. 0,13), mientras la escala de flexibilidad ideal $(\mathrm{N}=100)$ mostró correlaciones policóricas entre $-0,09$ y 0,59 (media 0,19 y D.E. 0,19 ). Las correlaciones ítem-resto para la escala de flexibilidad real estuvieron entre 0,15 y 0,35 (media 0,24 y D.E. 0,09), mientras que para la escala de cohesión ideal estuvieron entre 0,15 y 0,48 (media 0,32 y D.E. 0,12). Las matrices de correlación policórica ítemitem para escalas reales fueron semi-definidas positivas y se utilizaron para el siguiente análisis factorial.

Los resultados del AFE (factores principales) para cada escala por separado (cohesión y flexibilidad reales) se muestran en la Tabla 1. Allí se observan dos (2) factores retenidos para cohesión y tres (3) para flexibilidad, elegidos a partir de la inspección de los gráficos de sedimentación (Anexo 6). Las cargas factoriales mostradas se obtuvieron luego de una rotación Oblimin, mostrando bidimensionalidad para cohesión y tridimensionalidad para flexibilidad. La diferencia entre el primer y segundo autovalor es más alta para cohesión $(\Delta=3,3)$ que para flexibilidad $(\Delta=0,65)$, lo mismo que las comunalidades. Se verificó el supuesto de esfericidad (test de Bartlett, $p<0,001$ para ambas escalas) y la adecuación muestral $(\mathrm{KMO}=0,91$ para cohesión; $\mathrm{KMO}=0,68$ para flexibilidad).

Tabla 1. Cargas factoriales, autovalores y comunalidades para las escalas de Cohesión y flexibilidad real del FACESIII en los adolescentes de la muestra $(\mathrm{N}=910)$

\begin{tabular}{|c|c|c|c|c|c|c|c|c|}
\hline \multicolumn{4}{|c|}{ Escala cohesión real } & \multicolumn{5}{|c|}{ Escala flexibilidad real } \\
\hline Ítem & $\begin{array}{c}\text { Carga } \\
\text { factorial } \\
\text { factor } 1\end{array}$ & $\begin{array}{c}\text { Carga } \\
\text { factorial } \\
\text { factor } 2\end{array}$ & Comunalidad & Ítem & $\begin{array}{c}\text { Carga } \\
\text { factorial } \\
\text { factor } 1\end{array}$ & $\begin{array}{c}\text { Carga } \\
\text { factorial } \\
\text { factor } 2\end{array}$ & $\begin{array}{c}\text { Carga } \\
\text { factorial } \\
\text { factor } 3\end{array}$ & Comunalidad \\
\hline 1 & 0,61 & 0,00 & 0,37 & 2 & 0,62 & 0,07 & $-0,05$ & 0,43 \\
\hline 9 & 0,69 & 0,12 & 0,50 & 4 & 0,62 & 0,04 & $-0,05$ & 0,42 \\
\hline 11 & 0,77 & 0,11 & 0,60 & 10 & 0,60 & $-0,04$ & 0,08 & 0,34 \\
\hline 13 & 0,70 & 0,16 & 0,52 & 16 & 0,43 & $-0,15$ & 0,01 & 0,15 \\
\hline 15 & 0,62 & 0,08 & 0,39 & 6 & $-0,12$ & 0,38 & 0,05 & 0,14 \\
\hline 17 & 0,66 & 0,05 & 0,44 & 8 & 0,04 & 0,34 & $-0,04$ & 0,12 \\
\hline 19 & 0,72 & 0,12 & 0,53 & 12 & 0,14 & 0,41 & 0,06 & 0,26 \\
\hline 3 & 0,23 & 0,55 & 0,36 & 14 & 0,08 & 0,33 & 0,10 & 0,17 \\
\hline 5 & 0,01 & 0,82 & 0,66 & 18 & $-0,02$ & 0,05 & 0,56 & 0,34 \\
\hline 7 & 0,33 & 0,49 & 0,35 & 20 & 0,01 & $-0,02$ & 0,54 & 0,28 \\
\hline Autovalor & 3,50 & 0,20 & - & Autovalor & 1,54 & 0,89 & 0,22 & - \\
\hline Varianza & 3,48 & 1,88 & - & Varianza & 1,48 & 0,98 & 0,80 & - \\
\hline
\end{tabular}

Nota. Análisis factorial exploratorio realizado con el método de factores principales y rotación Oblimin. La elección de la cantidad de factores retenidos se hizo mediante la revisión del gráfico de sedimentación. Las cifras en negrita indican las cargas factoriales más altas. Las varianzas se han obtenido a partir de la rotación realizada. 
Tabla 2. Indicadores de bondad de ajuste de los modelos original y truncados para el FACES-III escalas reales en la muestra total de adolescentes $(\mathrm{N}=910)$

\begin{tabular}{|c|c|c|c|c|c|c|c|c|c|c|}
\hline Modelo & $\begin{array}{l}\text { Ítems } \\
\text { cohesión }\end{array}$ & Ítems flexibilidad & gl & $x^{2}$ & $\mathrm{X}^{2 / g 1}$ & AGFI & ECVI & NFI & GFI & RMSEA \\
\hline Original & $\begin{array}{c}\text { todos los } \\
\text { items impares }\end{array}$ & todos los ítems pares & 169 & $704,61^{*}$ & 4,17 & 0,96 & 0,87 & 0,93 & 0,97 & 0,059 \\
\hline Truncado 1 & ídem & ítems pares menos 12 y 18 & 134 & $403,70^{*}$ & 3,01 & 0,98 & 0,53 & 0,96 & 0,98 & 0,047 \\
\hline Truncado 2 & ídem & ítems pares menos 18 y 20 & 134 & $481,23^{*}$ & 3,59 & 0,97 & 0,61 & 0,95 & 0,98 & 0,053 \\
\hline Truncado 3 & ídem & ítems pares menos 6,18 y 20 & 118 & $399,59^{*}$ & 3,39 & 0,98 & 0,52 & 0,96 & 0,98 & 0,051 \\
\hline Truncado 4 & ídem & ítems pares menos 12,18 y 20 & 118 & $369,10^{*}$ & 3,13 & 0,98 & 0,48 & 0,96 & 0,98 & 0,048 \\
\hline Truncado 5 & ídem & ítems pares menos $6,12,18$ y 20 & 103 & $315,15^{*}$ & 3,06 & 0,98 & 0,42 & 0,97 & 0,99 & 0,048 \\
\hline Truncado 6 & ídem & $\begin{array}{l}\text { ítems pares menos } 6,8,12,14 \text {, } \\
18 \text { y } 20\end{array}$ & 76 & $215,36^{*}$ & 2,83 & 0,99 & 0,30 & 0,98 & 0,99 & 0,045 \\
\hline
\end{tabular}

$\mathrm{AGFI}=$ índice ajustado de bondad de ajuste. $\mathrm{ECVI}$ = índice de validación cruzada esperada. $\mathrm{NFI=índice} \mathrm{de} \mathrm{ajuste} \mathrm{normado.} \mathrm{GFI=índice}$ de bondad de ajuste. RMSEA = raíz del error cuadrático medio de aproximación; $\mathrm{X}^{2}$ = chi cuadrado escalada de Satorra-Bentler.

* $p<0,001$

Los resultados del AFC son visibles en la Tabla 2. Allí se aprecia cómo el primer modelo truncado es igual al original menos dos de los ítems que más afectaban la consistencia de la escala de flexibilidad (12 y 18). De esta forma, para cada modelo truncado se fueron retirando paulatinamente ítems heterogéneos (según se aprecia en dicha tabla) hasta llegar al sexto modelo truncado, en el cual se retiraron todos los ítems heterogéneos $(6,8,12,14,18$ y 20). La bondad de ajuste de estos modelos truncados (Tabla 2 ) es variable y se incrementa en la medida que se retiran ítems poco homogéneos. La figura 2 muestra los coeficientes estandarizados del modelo de medida del FACES-III original (con sus 20 ítems completos).

Los resultados del análisis de confiabilidad para el modelo original del FACES-III, según los índices alfa, theta y omega, pueden verse en la Tabla 3. Las escalas de cohesión, real e ideal, mostraron niveles altos de confiabilidad (consistencia interna), nunca menores a 0,79 . Las escalas de flexibilidad, real e ideal, mostraron niveles de confiabilidad moderados, entre 0,55 y 0,86 . En el Anexo 5 (material suplementario) puede verse una información similar (solo alfas de Cronbach), pero para los modelos truncados.

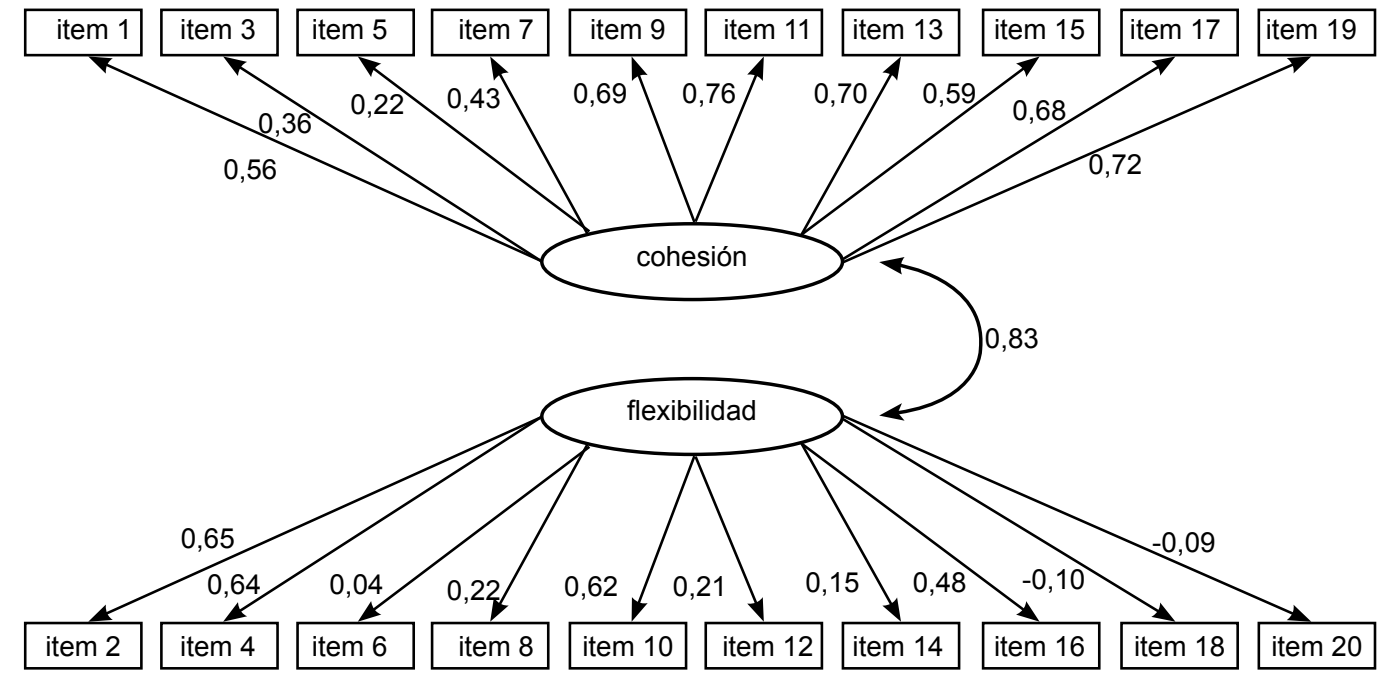

Nota. Los constructos latentes se muestran dentro de elipses y los reactivos se presentan en los rectángulos. Todos los coeficientes son significativos con una $\mathrm{p}<.05$, salvo los ítems 6 y 20 . Fuente: elaboración propia.

Figura 2. Coeficientes estandarizados para el modelo original del FACES-III en la muestra total de adolescentes $(\mathrm{N}=910)$ 
Tabla 3. Confiabilidad y descriptivos de las escalas cohesión y flexibilidad del FACES-III, real $(N=910)$ e ideal $(N=100)$ en adolescentes

\begin{tabular}{lccccc}
\hline & \multicolumn{2}{c}{ Real } & & \multicolumn{2}{c}{ Ideal } \\
\cline { 2 - 3 } \cline { 5 - 6 } & Cohesión & Flexibilidad & & Cohesión & Flexibilidad \\
\hline Alfa de Cronbach & 0,79 & 0,55 & 0,84 & 0,65 \\
Omega AFE & 0,85 & 0,74 & 0,89 & 0,86 \\
Theta AFE & 0,81 & 0,60 & 0,86 & 0,69 \\
Media & 37,7 & 28,7 & 40,7 & 31,1 \\
Desviación estandar & 6,8 & 5,6 & 7,2 & 6,5 \\
\hline
\end{tabular}

AEF: Análisis Factorial Exploratorio

\section{DISCUSIÓN}

El análisis de consistencia interna mostró que la escala cohesión-real tiene confiabilidad moderadamente alta $(\Omega=0,85)$, la escala flexibilidad-real tiene confiabilidad moderada $(\Omega=0,74)$, la escala cohesión-ideal tiene confiabilidad moderadamente alta $(\Omega=0,89)$, lo mismo que la escala de flexibilidad-ideal $(\Omega=0,86)$. También se analizó la confiabilidad de los modelos truncados propuestos para el AFC y se determinó que el modelo truncado 1 mejora la consistencia interna, retirando solo dos ítems (12 y 18). Una modificación del FACES-III a este nivel podría mejorar la confiabilidad sin sacrificar demasiado la validez de contenido; sin embargo, nos alejaría de su estándar de medida internacional, importante para la comparación de estudios transculturales en la línea de investigación de Olson. De hecho, en otros contextos una evidencia similar no ha propiciado la modificación de la escala de flexibilidad. Por ejemplo, véase cómo en España, donde la escala de cohesión tuvo un alpha $=0,72$ y la escala de flexibilidad un alpha $=0,68$ (27); o en Argentina, donde la de cohesión tuvo un alpha $=0,82$ y la de flexibilidad un alpha $=0,60$, no se generaron versiones recortadas del FACES-III (basadas en reducción de ítems heterogéneos). En tal sentido, la confianza en la precisión del FACES-III será una decisión del usuario final ${ }^{(28)}$ quien deberá tener presente otros aspectos, como el uso que le dará al test (v.g. evaluación grupal o individual) ${ }^{(29)}$, antes de elegirlo como herramienta de evaluación.

La evidencia de unidimensionalidad es moderada para la escala de cohesión-real y baja para flexibilidad real. El AFE para la escala de cohesión mostró hasta dos dimensiones; no obstante, un índice theta alto $(\theta=0,81)$ señal de unidimensionalidad coherente con lo hallado en estudios previos ${ }^{(3,12,27,30)}$. Por otro lado, el AFE para flexibilidad-real sugirió hasta tres dimensiones, con un índice theta moderado $(\theta=0,60)$, lo que resta en contra de la unidimensionalidad del constructo. De hecho, la evidencia antecedente sobre flexibilidad es contradictoria. Un estudio en población Argentina sugiere una estructura bidimensional para la flexibilidad, desde la evidencia de un análisis factorial confirmatorio (12); otro estudio en adolescentes de zonas rurales norteamericanas avala la unidimensionalidad de la escala de flexibilidad solo para 7 de los 10 ítems, trasladando los otros 3 a la escala de cohesión (30); y un riguroso estudio español apunta hacia la unidimensionalidad de esta escala, con todos los ítems ${ }^{(10)}$, pero violando el supuesto de varianzas de error incorrelacionadas para los pares de ítems 1-17, 9-15 y 11-19 en el contexto de un análisis factorial confirmatorio ${ }^{(27)}$. El origen de estas diferencias puede radicar en características intrínsecas a cada población, o en las herramientas estadísticas utilizadas (exploratorias o confirmatorias) o en la forma en que fueron aplicadas (evaluación de las escalas de cohesión y flexibilidad por separado o en un mismo modelo confirmatorio), o en todas ellas. Lo cierto es que soluciones de más de un factor no tienen cabida en el modelo circumplejo original de Olson (3): la flexibilidad es una y si emergen nuevas dimensiones de su escala, estas solo cobrarían sentido en dicho modelo si lograran encajar en alguna de las ya teorizadas (por ejemplo, comunicación o satisfacción). Ciertamente, esto no está ocurriendo, pues los ítems de flexibilidad fueron concebidos en base a otros indicadores: liderazgo, disciplina, control y roles-reglas.

Todos los modelos truncados presentan mejor bondad de ajuste que el modelo original; sin embargo, este último mantiene una bondad de ajuste suficiente sin sacrificar validez de contenido. Así, para el modelo original véase que (Tabla 2): el índice de bondad de ajuste GFI tiene un valor alto $(0,97)$, el RMSEA está por debajo del 0,08 recomendado como límite ${ }^{(31)}$, el ajuste incremental NFI de 0,93 está por encima del nivel recomendado de $0,90{ }^{(32)} \mathrm{y}$, aunque la chi cuadrado normada es alta $(4,17)$, el índice ajustado de bondad de ajuste AGFI tiene un valor alto $(0,96)$, lo que es buena señal de la parsimonia del modelo. Los modelos truncados mejoran varios de estos indicadores, pero sacrificando ítems. Solo el modelo truncado 1 produce una mejora importante con un mínimo de pérdida (dos ítems: 12 y 18). Para un usuario local que desee optimar la escala de flexibilidad sin atentar demasiado contra la validez de contenido del FACES-III original, resulta plausible trabajar con este modelo, siempre que tenga en cuenta las implicancias de una operación como esta (v.g. imposibilidad de usar una norma original o local basada en todos los ítems). 
En este punto de la discusión es necesario retomar un problema antiguo a la luz de la nueva evidencia: el FACES-III no realiza mediciones ortogonales en nuestra población, ¿qué significa esto?, que las mediciones de cohesión y flexibilidad no están incorrelacionadas entre sí, lo que es un supuesto elemental del modelo circumplejo de Olson. Explicando la historia (3): el FACES-II ya tenía este problema y, por ello, el proceso de construcción del FACES-III giró en torno a una selección de ítems que permitiera cumplir con dicho supuesto teórico (reducción de ítems hasta llegar a los 20 que hoy conocemos). En principio, Olson lo logró ( $r=0,03$ entre ambas escalas) y esto funcionó más o menos bien en otros contextos (v.g. un $\beta=0,28$ en el estudio de Schmidt et al. ${ }^{\left({ }^{12}\right)}$ ). Sin embargo, en nuestro estudio esta propiedad no se ha confirmado: i) la correlación simple entre ambas escalas es de $r=0,44$ y ii) la señal de correlación más limpia, proveniente del $A F C$, señala una relación aún mayor $(\beta=0,83)$. Esto tiene repercusiones directas en las interpretaciones de los resultados; o sea, no se discute la bidimensionalidad del constructo funcionalidad familiar, pero sí la ortogonalidad de sus dimensiones. Para efectos prácticos, se dificulta la construcción de los 16 tipos familiares y calzarlos en los tres niveles de funcionalidad deja de estar empíricamente justificado, ¿qué se puede recomendar en este punto?: trabajar la interpretación de funcionalidad (balanceada, extrema y media) directamente sobre las dimensiones por separado, y no sobre los tipos familiares. Por supuesto, esta no es una solución final, pero puede ser de ayuda para cualquier fin evaluativo inmediato.

Hemos identificado al menos una limitación y una fortaleza del presente estudio. La revisión de validez y confiabilidad del instrumento pudo haber sido más completa y efectiva; especialmente si se hubiese incorporado la evaluación de todos los actores (padres, hijos y demás miembros de la familia). Así, la evaluación psicométrica del FACES-III aquí realizada se limita a la percepción de solo uno de los miembros de la familia: el hijo adolescente. No obstante, Olson ha habilitado esta como una opción plausible de evaluación ${ }^{(3)}$ que, de hecho, ya se ha usado anteriormente en otros estudios psicométricos ${ }^{(13)}$. En tal sentido, los resultados aquí obtenidos son valiosos para los usuarios locales que -igualmente - realizan evaluaciones de funcionalidad familiar con base en la sola percepción del hijo adolescente ${ }^{(33-35)}$. Por otro lado, una fortaleza del estudio es su adecuado tamaño muestral. La muestra grande nos ha permitido confirmar que la cohesión es un constructo de medición robusta y que la flexibilidad debe leerse con más cuidado. Además, ha permitido constatar que la interpretación de tipos familiares no es consistente en nuestro contexto, pero que la funcionalidad familiar sí puede interpretarse separadamente para cada dimensión (cohesión y adaptabilidad).

En suma, la evidencia presentada en este estudio permite concluir que el FACES-III tiene confiabilidad y validez suficientes como para ser utilizado en población adolescente peruana con fines de evaluación grupal o individual.

Contribuciones de los autores: JCBA, OABA, JA, FP, WM e IB han participado en la concepción y diseño del artículo, su redacción y aprobación de la versión final. Además, JCBA y JA participaron en la recolección de datos. JCBA realizó los análisis estadísticos y obtuvo el financiamiento.

Fuentes de financiamiento: financiamiento del Instituto de Investigación y Capacitación para la Prevención y Promoción Psicoeducativa y Psicosocial - PSYCOPERU.

Conflictos de interés: los autores declaran no tener conflictos de interés en la publicación del artículo.

Anexos disponibles en: http://www.rpmesp.ins.gob.pe/index. php/rpmesp/article/view/2299/2212

\section{REFERENCIAS BIBLIOGRÁFICAS}

1. Olson DH, Bell R, Portner J. FACES: Family Adaptability and Cohesion Evaluation Scales (manual). St. Paul: University of Minnesota; 1978.

2. Olson DH, Portner J, Bell R. FACES II: Family adaptability and cohesion evaluation scales. Minnesota: Family Social Science, University of Minnesota; 1982.

3. Olson DH, Portner J, Lavee Y. Family Adaptability and Cohesion Evaluation Scales III (FACES III). Minnesota: University of Minnesota; $1985.180 \mathrm{p}$.

4. Olson DH. FACES III: Family Social Science, University of Minnesota; 1985.
5. Olson DH, Tiesel J, Gorall D, Fitterer C. Family assessment package. Unpublished Manuscript, University of Minnesota, St Paul. St Paul: University of Minnesota; 1996.

6. Gorall D, Tiesel J, Olson D. FACES IV: Development and validation. Minneapolis, MN: Life Innovations; 2004

7. Olson DH, Gorall DM, Tiesel J. FACES IV \& the circumplex model. Minneapolis, MN: Life Innovations, Inc; 2006.

8. Olson DH. FACES IV and the Circumplex Model: Validation study. J Marital Fam Ther. 2011;37(1):64-80. doi: $10.1111 /$ j.1752-0606.2009.00175.x.
9. Olson DH. Circumplex model VII: Validation studies and FACES III. Fam Process. 2004;25(3):337-51.

10. Polaino-Lorente A, Cano PM. Evaluación psicológica y psicopatológica de la familia. Madrid: Ediciones Rialp S.A.; 1998.

11. Martínez-Pampliega A, Iraugi I, Sanz M. Validez estructural del FACES 20-Esp: versión española de 20 ítems de la escala de evaluación de la cohesión y adaptabilidad familiar. RIDEP. 2011;29(1):147-65.

12. Schmidt V, Barreyro JP, Maglio AL. Escala de evaluación del funcionamiento familiar FACES III: ¿Modelo de dos 
o tres factores? Escritos de Psicología. 2010;3(2):30-6

13. Ponce Rosas ER, Gómez Clavelina $\mathrm{FJ}$, Terán Trillo $\mathrm{M}$, Irigoyen Coria AE, Landgrave Ibáñez S. Validez de constructo del cuestionario FACES III en español (México). Aten Primaria. 2002;30(10):624-30.

14. Zegers B, Larraín ME, Polaino-Lorente A, Trapp A, Diez I. Validezy confiabilidad de la versión española de la escala de Cohesión y Adaptabilidad Familiar (CAF) de Olson, Russell \& Sprenkle para el diagnóstico del funcionamiento familiar en la población chilena. Rev Chil Neuro-Psiquiat. 2003;41(1):39-54. doi: http://dx.doi.org/10.4067/S071792272003000100006

15. Vandeleur CL, Preisig M, Fenton BT, Ferrero F. Construct validity and internal reliability of a French version of FACES III in adolescents and adults. Swiss journal of psychology. 1999;58(3):161-9. doi: http://dx.doi.org/10.1024//14210185.58.3.161

16. MacCallum RC, Widaman KF, Zhang $S$, Hong S. Sample size in factor analysis. Psychol Methods. 1999;4(1):84.

17. MacCallum RC, Widaman KF, Preacher KJ, Hong S. Sample size in factor analysis: The role of model error. Multivariate Behav Res. 2001;36(4):611-37. doi: 10.1207/S15327906MBR3604_06.

18. Maxwell SE, Kelley K, Rausch JR. Sample size planning for statistical power and accuracy in parameter estimation. Annu Rev Psychol. 2008;59:537-63.

19. MacCallum RC, Browne MW, Cai L. Testing differences between nested covariance structure models: Power analysis and null hypotheses. Psychol Methods. 2006;11(1):19-35.
20. MacCallum RC, Browne MW, Sugawara HM. Power analysis and determination of sample size for covariance structure modeling. Psychol Methods. 1996;1(2):130-49.

21. MacCallum RC, Hong S. Power analysis in covariance structure modeling using GFI and AGFI. Multivariate Behav Res. 1997;32(2):193-210. doi: 10.1207/ s15327906mbr3202_5.

22. $\mathrm{Kim} \mathrm{KH}$. The relation among fit indexes, power, and sample size in structural equation modeling. Structural Equation Modeling. 2005;12(3):368-90. doi: $10.1207 /$ s15328007sem1203_2.

23. Polaino Lorente A, Martinez Cano P. Versión española de Family Adaptability and Cohesion Evaluation Scales III (FACES III) de Olson, H. Portier, J. 1998.

24. Santisteban C. Principios de psicometría. Madrid: Editorial Síntesis S.A. 2009.

25. Beauducel A, Herzberg PY. On the performance of maximum likelihood versus means and variance adjusted weighted least squares estimation in CFA. Structural Equation Modeling. 2006;13(2):186-203. doi: 10.1207/ s15328007sem1302_2

26. Brown TA. Confirmatory factor analysis for applied research. New York: Guilford Press; 2015.

27. Forjaz MJ, Cano PM, Cervera-Enguix S. Confirmatory factor analysis, reliability, and validity of a Spanish version of FACES III. Am J Fam Ther. 2002;30(5):439-49. Doi: $10.1080 / 01926180260296332$.

28. American Educational Research Association, American Psychological Association, and the National Council on Measurement in Education. Standards for educational and psychological testing. Washington, D.C.: AERA; 1999.

29. Aiken LR. Tests psicológicos y evaluación. México: Pearson Educación; 2003.

30. Ide B, Dingmann C, Cuevas E, Meehan M. Psychometric testing of the FACES III with rural adolescents. J Fam Soc Work. 2010;13(5):410-9. doi: $10.1080 / 10522150903513993$.

31. Browne MW, Cudeck R, Bollen KA, Long JS. Alternative ways of assessing model fit. Sage Focus Editions. 1993;154:136.

32. Hair J, Anderson R, Tatham R, Black W. Análisis Multivariante. Londres: Prentice Hall; 1999.

33. Muñoz J, Pinto V, Callata H. Ideación suicida y cohesión familiar en estudiantes preuniversitarios entre 15 y 24 años, Lima 2005. Rev Peru Med Exp Salud Publica. 2006;23(4):239-46.

34. Camacho Palomino P, León Nakamura CL, Silva Mathews I. Funcionamiento familiar según el Modelo Circumplejo de Olson en adolescentes. Rev Enferm Herediana. 2009;2(2):80-5.

35. Cárdenas R. Cohesión y adaptabilidad familiar y su relación con el rendimiento académico [Tesis]. Lima: Universidad Nacional Mayor de San Marcos; 2004.

Correspondencia: Juan Carlos Bazo-Álvarez. Dirección: Departamento Académico de Estadistica, Demografia, Humanidades y Ciencias Sociales, Facultad de Ciencias y Filosofía Alberto Cazorla Talleri, Universidad Peruana Cayetano Heredia. Av. Honorio Delgado 430, Urb. Ingeniería, San Martín de Porres, Lima, Perú.

Correo electrónico:juan.bazo.a@upch.pe 


\section{ANEXOS}

Anexo 1. Cantidad de unidades en la muestra según estratos y conglomerados

\begin{tabular}{|c|c|c|c|c|c|c|c|c|}
\hline \multirow[b]{2}{*}{ Institución educativa y año } & \multicolumn{7}{|c|}{ Sección } & \multirow[b]{2}{*}{$\mathrm{N}=910$} \\
\hline & A & B & C & D & $\mathbf{E}$ & $\mathbf{F}$ & G & \\
\hline \multicolumn{9}{|l|}{ Institución Educativa Nacional } \\
\hline Primero secundaria & 39 & 0 & 0 & 37 & 0 & 0 & 38 & 114 \\
\hline Segundo secundaria & 0 & 37 & 0 & 0 & 38 & 36 & 0 & 111 \\
\hline Tercero secundaria & 32 & 0 & 41 & 39 & 0 & 0 & 0 & 112 \\
\hline Cuarto secundaria & 0 & 37 & 0 & 0 & 38 & 36 & 0 & 111 \\
\hline Quinto secundaria & 0 & 0 & 34 & 32 & 30 & 0 & 0 & 96 \\
\hline Total & 71 & 74 & 75 & 108 & 106 & 72 & 38 & 544 \\
\hline \multicolumn{9}{|c|}{ Institución Educativa particular } \\
\hline Sexto primaria & 24 & 22 & 0 & - & - & - & - & 46 \\
\hline Primero secundaria & 29 & 26 & 27 & - & - & - & - & 82 \\
\hline Segundo secundaria & 27 & 25 & 26 & - & - & - & - & 78 \\
\hline Tercero secundaria & 30 & 28 & 0 & - & - & - & - & 58 \\
\hline Cuarto secundaria & 0 & 34 & 0 & - & - & - & - & 34 \\
\hline Quinto secundaria & 24 & 23 & 21 & - & - & - & - & 68 \\
\hline Total & 134 & 158 & 74 & - & - & - & - & 366 \\
\hline
\end{tabular}


Anexo 2. Matriz de operacionalización del FACES - III propuesta por los autores

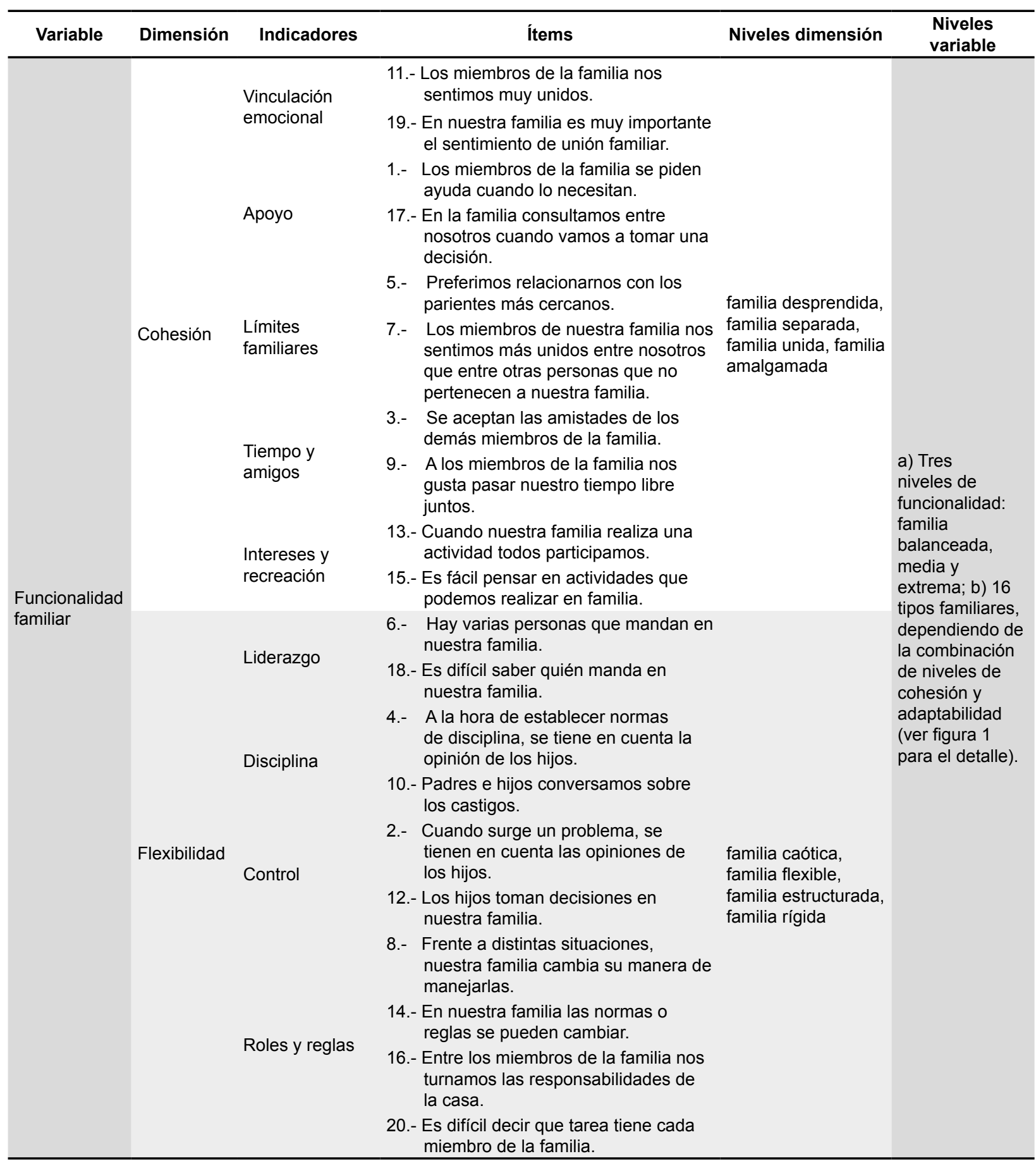


Anexo 3. Descriptivos antes y después de la imputación de datos de la escala de cohesión "real" en adolescentes de Chimbote 2014

\begin{tabular}{|c|c|c|c|c|c|c|c|c|}
\hline \multirow{2}{*}{ Ítem } & \multicolumn{4}{|c|}{$\begin{array}{l}\text { Antes de imputar } \\
(\mathrm{N}=910)\end{array}$} & \multicolumn{4}{|c|}{$\begin{array}{l}\text { Después de imputar } \\
(\mathrm{N}=910)\end{array}$} \\
\hline & media & d.e. & $\begin{array}{c}\text { valores } \\
\text { perdidos (n) }\end{array}$ & $\begin{array}{c}\text { valores } \\
\text { perdidos (\%) }\end{array}$ & media & d.e. & $\begin{array}{l}\text { valores } \\
\text { perdidos (n) }\end{array}$ & $\begin{array}{c}\text { valores } \\
\text { perdidos (\%) }\end{array}$ \\
\hline 1 & 3,7 & 1,1 & 3 & 0,33 & 3,7 & 1,1 & 0 & 0,00 \\
\hline 2 & 3,2 & 1,2 & 7 & 0,77 & 3,2 & 1,2 & 0 & 0,00 \\
\hline 3 & 3,9 & 1,1 & 4 & 0,44 & 3,9 & 1,1 & 0 & 0,00 \\
\hline 4 & 3,3 & 1,2 & 3 & 0,33 & 3,3 & 1,2 & 0 & 0,00 \\
\hline 5 & 3,6 & 1,1 & 6 & 0,66 & 3,6 & 1,1 & 0 & 0,00 \\
\hline 6 & 2,6 & 1,4 & 13 & 1,43 & 2,6 & 1,4 & 0 & 0,00 \\
\hline 7 & 3,8 & 1,2 & 10 & 1,10 & 3,8 & 1,2 & 0 & 0,00 \\
\hline 8 & 3,2 & 1,1 & 8 & 0,88 & 3,2 & 1,1 & 0 & 0,00 \\
\hline 9 & 3,5 & 1,3 & 6 & 0,66 & 3,5 & 1,3 & 0 & 0,00 \\
\hline 10 & 2,9 & 1,3 & 8 & 0,88 & 2,9 & 1,3 & 0 & 0,00 \\
\hline 11 & 4,0 & 1,1 & 16 & 1,76 & 4,0 & 1,1 & 0 & 0,00 \\
\hline 12 & 2,2 & 1,2 & 16 & 1,76 & 2,2 & 1,2 & 0 & 0,00 \\
\hline 13 & 4,0 & 1,1 & 8 & 0,88 & 4,0 & 1,1 & 0 & 0,00 \\
\hline 14 & 2,8 & 1,2 & 21 & 2,31 & 2,8 & 1,2 & 0 & 0,00 \\
\hline 15 & 3,5 & 1,1 & 18 & 1,98 & 3,5 & 1,1 & 0 & 0,00 \\
\hline 16 & 3,6 & 1,3 & 9 & 0,99 & 3,6 & 1,3 & 0 & 0,00 \\
\hline 17 & 3,5 & 1,2 & 6 & 0,66 & 3,5 & 1,2 & 0 & 0,00 \\
\hline 18 & 2,3 & 1,4 & 6 & 0,66 & 2,3 & 1,4 & 0 & 0,00 \\
\hline 19 & 4,2 & 1,1 & 4 & 0,44 & 4,2 & 1,1 & 0 & 0,00 \\
\hline 20 & 2,5 & 1,3 & 7 & 0,77 & 2,5 & 1,3 & 0 & 0,00 \\
\hline
\end{tabular}

d.e.: desviación estándar

Anexo 4. Descriptivos antes y después de la imputación de datos de la escala de cohesión "ideal" en adolescentes de Chimbote 2014

\begin{tabular}{|c|c|c|c|c|c|c|c|c|}
\hline \multirow{2}{*}{ Ítem } & \multicolumn{4}{|c|}{$\begin{array}{l}\text { Antes de imputar } \\
(\mathrm{N}=100)\end{array}$} & \multicolumn{4}{|c|}{$\begin{array}{c}\text { Después de imputar } \\
(\mathrm{N}=100)\end{array}$} \\
\hline & media & d.e. & $\begin{array}{l}\text { valores } \\
\text { perdidos (n) }\end{array}$ & $\begin{array}{c}\text { valores } \\
\text { perdidos (\%) }\end{array}$ & media & d.e. & $\begin{array}{l}\text { valores } \\
\text { perdidos }(n)\end{array}$ & $\begin{array}{c}\text { valores } \\
\text { perdidos (\%) }\end{array}$ \\
\hline 1 & 4,2 & 1,0 & 0 & 0,00 & 4,2 & 1,0 & 0 & 0,00 \\
\hline 2 & 3,8 & 1,2 & 0 & 0,00 & 3,8 & 1,2 & 0 & 0,00 \\
\hline 3 & 3,9 & 1,2 & 0 & 0,00 & 3,9 & 1,2 & 0 & 0,00 \\
\hline 4 & 3,8 & 1,2 & 0 & 0,00 & 3,8 & 1,2 & 0 & 0,00 \\
\hline 5 & 3,5 & 1,4 & 1 & 1,00 & 3,3 & 1,3 & 0 & 0,00 \\
\hline 6 & 2,4 & 1,5 & 2 & 2,00 & 2,4 & 1,5 & 0 & 0,00 \\
\hline 7 & 4,1 & 1,1 & 1 & 1,00 & 4,1 & 1,1 & 0 & 0,00 \\
\hline 8 & 3,2 & 1,4 & 0 & 0,00 & 3,2 & 1,4 & 0 & 0,00 \\
\hline 9 & 4,0 & 1,3 & 0 & 0,00 & 4,0 & 1,3 & 0 & 0,00 \\
\hline 10 & 3,6 & 1,3 & 1 & 1,00 & 3,5 & 1,1 & 0 & 0,00 \\
\hline 11 & 4,4 & 1,0 & 2 & 2,00 & 4,8 & 1,2 & 0 & 0,00 \\
\hline 12 & 2,5 & 1,3 & 2 & 2,00 & 2,8 & 1,2 & 0 & 0,00 \\
\hline 13 & 4,2 & 1,1 & 3 & 3,00 & 4,4 & 1,2 & 0 & 0,00 \\
\hline 14 & 2,9 & 1,3 & 1 & 1,00 & 3,2 & 1,4 & 0 & 0,00 \\
\hline 15 & 3,8 & 1,2 & 1 & 1,00 & 3,6 & 1,3 & 0 & 0,00 \\
\hline 16 & 4,0 & 1,2 & 0 & 0,00 & 4,0 & 1,2 & 0 & 0,00 \\
\hline 17 & 4,1 & 1,1 & 1 & 1,00 & 4,3 & 1,3 & 0 & 0,00 \\
\hline 18 & 2,4 & 1,4 & 7 & 7,00 & 2,0 & 1,2 & 0 & 0,00 \\
\hline 19 & 4,5 & 0,8 & 2 & 2,00 & 4,4 & 0,9 & 0 & 0,00 \\
\hline 20 & 2,6 & 1,4 & 0 & 0,00 & 2,6 & 1,4 & 0 & 0,00 \\
\hline
\end{tabular}

d.e.: desviación estándar 
Anexo 5. Alfa de Cronbach de las escalas cohesión y flexibilidad del Faces-III, real $(N=910)$ e ideal $(N=100)$, para los diferentes modelos propuestos

\begin{tabular}{lcccccc}
\hline & \multicolumn{2}{c}{ Real } & & \multicolumn{2}{c}{ Ideal } \\
\cline { 2 - 3 } \cline { 5 - 6 } & cohesión & flexibilidad & & cohesión & flexibilidad \\
\hline Original & 0,79 & 0,55 & & 0,84 & 0,65 \\
Truncado 1 & ídem & 0,49 & & ídem & 0,60 \\
Truncado 2 & ídem & 0,57 & & ídem & 0,65 \\
Truncado 3 & ídem & 0,60 & & ídem & 0,66 \\
Truncado 4 & ídem & 0,53 & & ídem & 0,59 \\
Truncado 5 & ídem & 0,58 & & ídem & 0,62 \\
Truncado 6 & ídem & 0,63 & & ídem & 0,70 \\
\hline
\end{tabular}

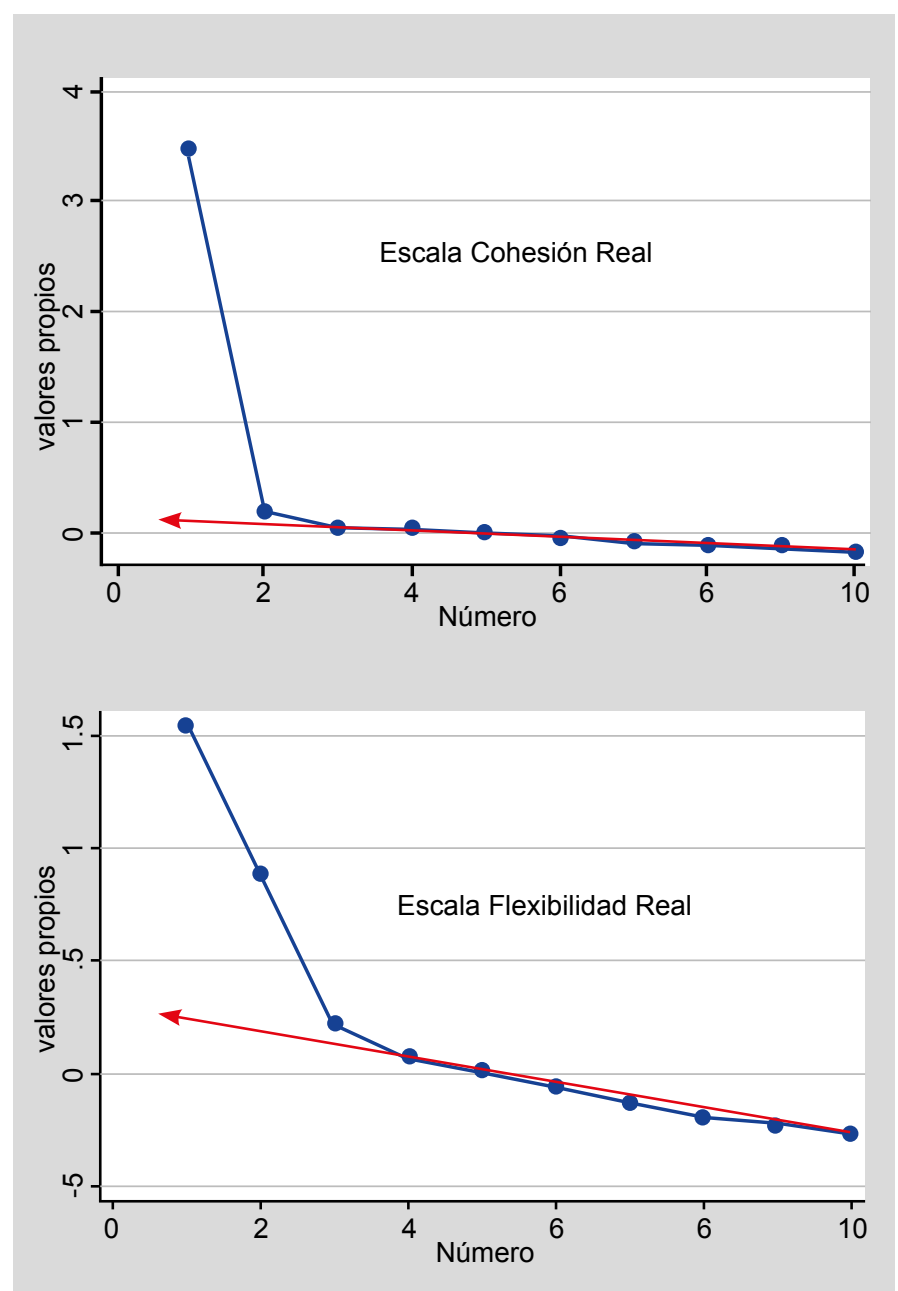

Anexo 6. Gráfico de sedimentación para la selección de factores de las escalas de flexibilidad y cohesión real. 